\title{
Design of a USB based Multichannel, Low Cost Data Acquisition System using PIC Microcontroller
}

\author{
Anindita Bora \\ Dept of Instrumentation \& USIC \\ Gauhati University \\ Guwahati - 781014, Assam, India
}

\author{
Kanak Chandra Sarma \\ Dept of Instrumentation \& USIC \\ Gauhati University \\ Guwahati - 781014, Assam, India
}

\begin{abstract}
This paper describes the design and development of a low cost Data Acquisition System (DAS) using PIC18LF4553 microcontroller for real time data acquisition. The designed DAS has 4 analog input channels having 12-bit resolution and was interfaced through the USB port of the PC. The interface to the PC is basically a USB based virtual serial port emulation using FT232R, a USB to serial UART interfacing IC. The PIC microcontroller firmware has been written in C language and compiled using MikroC compiler for PIC and downloaded to the microcontroller by using USBurn programmer for PIC. A PC application program has been also developed using MATLAB, which allows displaying the waveform of the signal(s) in real time and storing the data into the hard disk of the computer for future use and analysis.
\end{abstract}

\section{General Terms}

Data Acquisition System (DAS), Universal Serial Bus (USB), Microcontroller.

\section{Keywords}

Data Acquisition System, Microcontroller, USB to UART interface, Accelerometer.

\section{INTRODUCTION}

With the rapid development in the field of embedded technology there is also an increasing thrust for a data acquisition system which is fast in processing speed, small in size, low in cost and monitors the data in real time basis. The use of a microcontroller as a processor has become popular because of its speed, energy efficiency, low cost and low weight [1-2], which leads to the broad use of it in Data Acquisition System (DAS). Data Acquisition System is a system which is used for acquisition of signals of physical parameters continuously for a certain period of time and keeps a record of those acquired values for future use. A typical DAS has a primary control and data processor, memory and a clock /calendar module for time stamping the acquired data. A number of sensor attachments depending on the applications are also necessary. As most of the internal processors are digital in nature, hence the analog signals are often converted to digital format before being used for processing [1]. For analysis, display and recording, the processor is further connected with computer or Laptops. The complexity of a DAS system tends to increase with the increase in the number of physical properties to be measured, resolution of ADC and accuracy and speed of the measurement required. In this work, the design and development of a USB based DAS system using PIC18LF4553 has been presented. PIC 18LF4553 microcontroller is ideal for low power application. It has 13 channel 12 bit ADC and has three serial ports: USB,
SPI and an asynchronous serial port [3]. The USB based interface with computer is most favorable because of its high data transmission rate and ease of connectivity with computer. Hence the designed USB based multi channel DAS system can give a high computational performance at an economical price[4-6].

\section{HARDWARE DESIGN}

The block diagram of the proposed design is shown in the Fig 1. As an example the designed DAS system is used to monitor the values of an accelerometer. The designed circuit diagram along with the transducer is shown in the Fig 2. The details of the hardware, the firmware and the software parts for the real time monitoring DAS are described below.

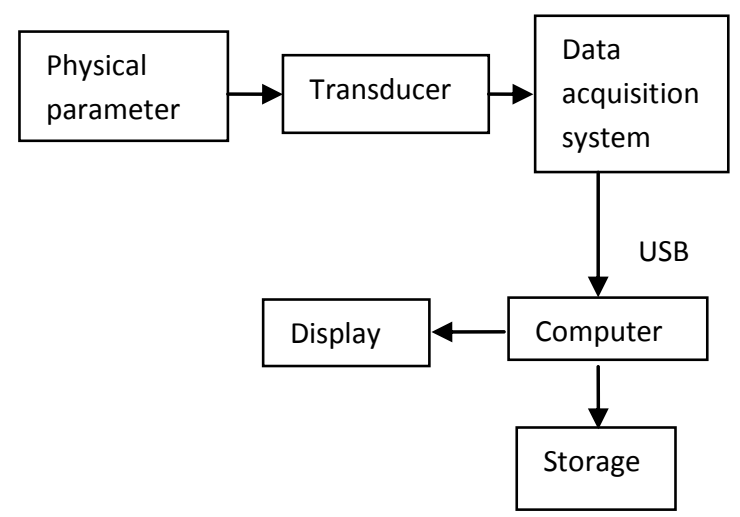

Fig 1: Block diagram of the designed Data Acquisition System

\subsection{Data Acquisition Unit}

The data acquisition system has been developed using PIC 18LF4553 which is a mid range 40 Pin, High-Performance, Enhanced Flash, USB Microcontrollers with 12-Bit A/D. The circuit is powered from the PC through USB cable. The PIC has 13 channels $\mathrm{ADC}$ with 12 bit resolution. The analog signals required to monitor is applied to the ADC input. As the $\mathrm{ADC}$ is of 12 bit resolution so it will read the signal in 4096 steps. The reference voltage applied to the ADC is 5 volt hence it has an accuracy of $1.22 \mathrm{mV}$. 


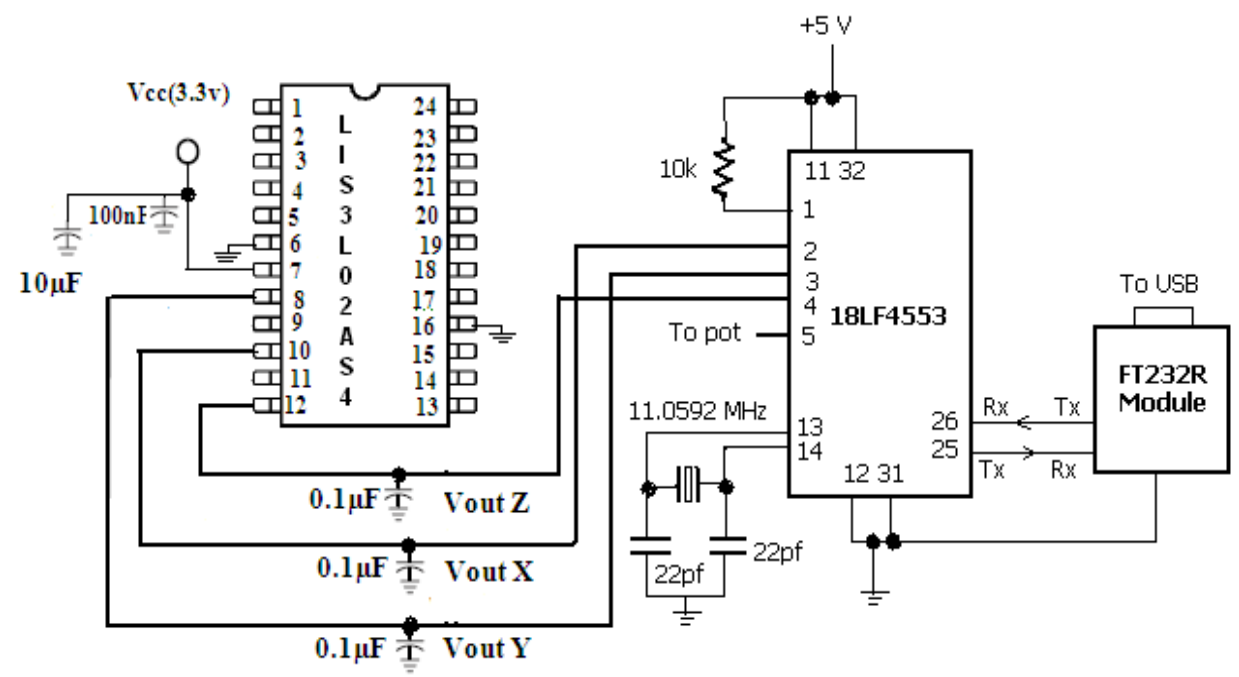

Fig 2: Circuit diagram of the designed Data Acquisition System along with sensor.

\subsection{Sensors}

The data so converted is transferred to the PC using FT232R, a USB to serial UART interfacing IC. The LIS3L02AS4 is a sensor capable of measuring tri-axis linear acceleration. It includes a sensing element and an IC interface which provides an analog signal equivalent to the measuring value of the acceleration to the outside world. It is capable of measuring accelerations over a maximum bandwidth of $4.0 \mathrm{KHz}$ for the $\mathrm{X}$ and $\mathrm{Y}$ axis and $2.5 \mathrm{KHz}$ for the $\mathrm{Z}$ axis. The IC interface is factory calibrated to provide to the final user a device ready to operate.

\subsection{Firmware}

The data is transferred to the pc using a USB to serial UART interfacing IC. The interface to the pc is thus USB. On the pc side the IC, FT232R emulates a virtual serial port [7]. On the DAS side the data is transferred to the UART of FT232R. Therefore on the DAS side the communication is through the UART of the microcontroller. The use of FT232R facilitates the advantage of USB portability and eliminates the need to write complex code for USB communication as the communication code is to be written only for UART. The PIC program is written in $\mathrm{C}$ language and compiled in mikroC to generate the Hex file. The hex file so generated is transferred to the PIC 18LF4553 microcontroller through In-Circuit Serial Programming (ICSP).

The UART baud rate has been set at 460800 to maximize the data transfer rate. The communication through UART is asynchronous. The PC application program initiates the data acquisition system. The PC request the DAS for data by sending a character ' $r$ ' through the UART. On receiving the character ' $r$ ' by the DAS, it reads the analog input channels and formats the digitized data in a string "Start12345123451234512345end", which is of length 28 character. The string is then sent asynchronously to the PC. In the string the character sequence "Start" represent the start of the string, the next five characters represent the decimal value of 12-bit ADC for channel 0. Similarly the next five characters represent data for channel 1 and so on. In this DAS system we have developed the code for 4 channel analog input. At the end of the $4^{\text {th }}$ channel data, the string is appended with character sequence "end" to represent the end of the string. The flow chart of the program so developed is shown in figure 3 .

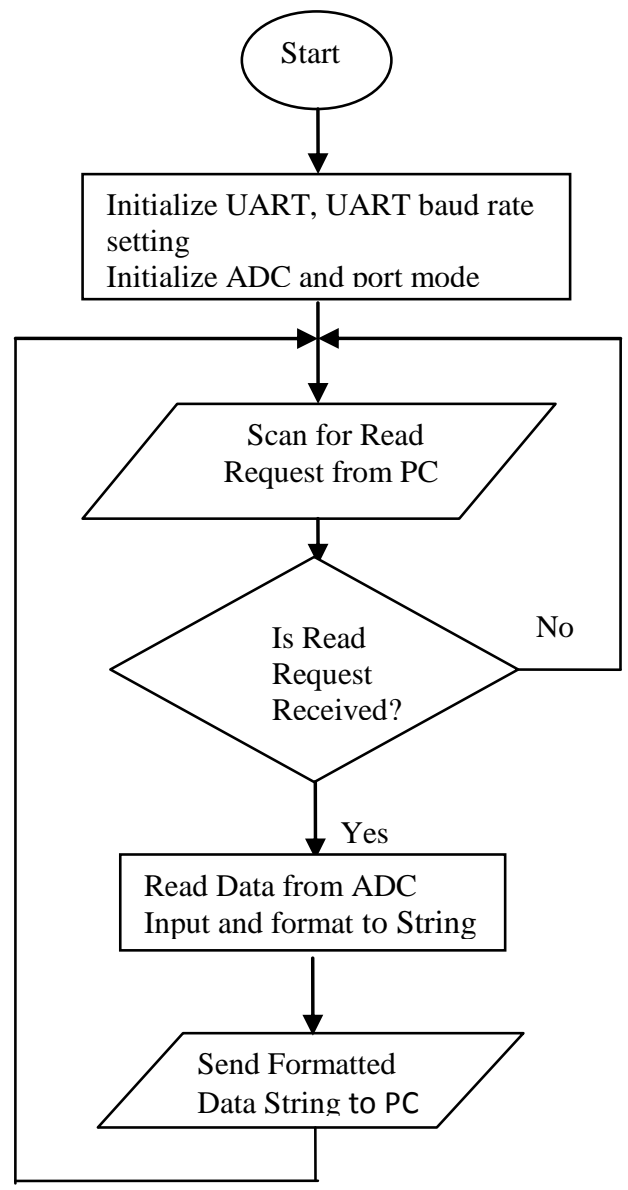

Fig 3: Flow chart of the firmware 


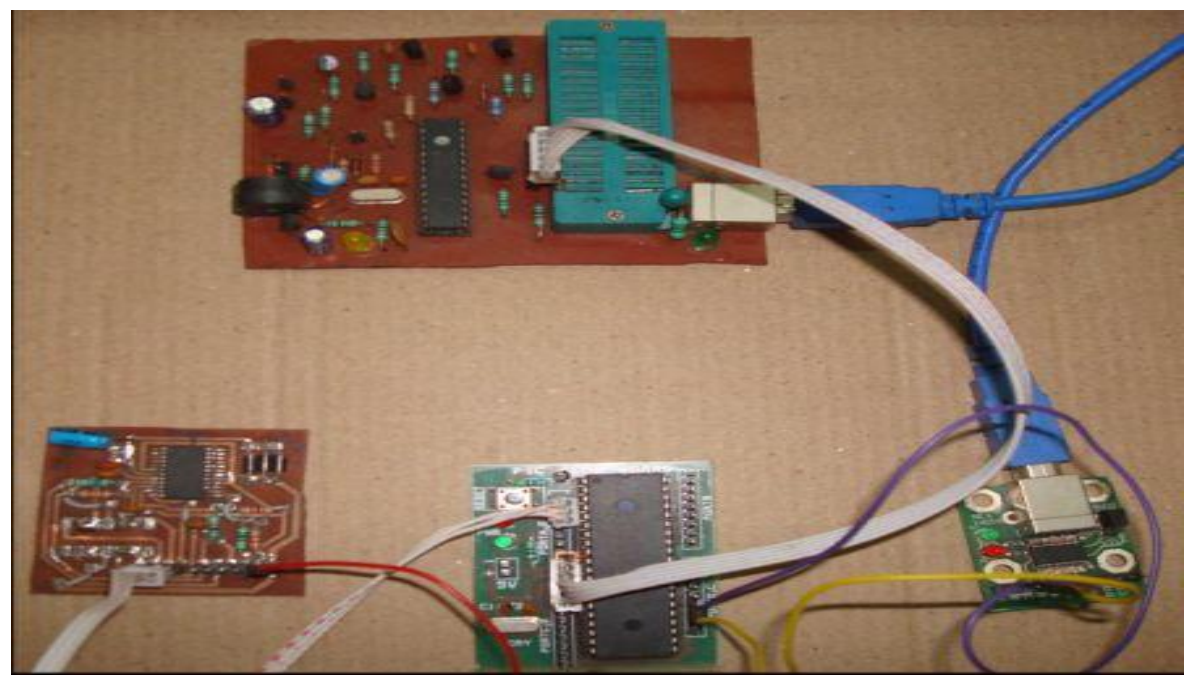

Fig 4: Photograph of the DAS along with sensor and programmer

\subsection{Application Program}

A PC application program is developed in Matlab for proper acquisition of the data, real-time graphical display and storing the acquired data into a file. The Application program accesses the USB device as a standard COM port device of the PC [8]. The application program is developed to acquire data for a user settable time duration. As the program is started it sends the read character ' $r$ ' to the DAS. The DAS then sends the data string to the pc. The application program then performs an error checking of the received data string. Herea simple error checking methodology has been applied. First the string is checked for its length, which has to be 28 . If the length is found correct then the character sequence "Start" and "end" at the beginning and end of the string is checked. If no error is found the data string is declared valid and processed to extract the analog voltage at all the channels. The analog voltage is then plotted in real-time and also stored in Matlab workspace. The application program then requests for another data string and the process continues for the time duration specified by the user. After the specified time duration of acquisition, the application program stores the acquired data available in workspace to a file on the pc environment for future use. In this case the output of an accelerometer module is connected to 3 channels of the ADC. The accelerometer is displaced along all the axis back and forth. The fourth channel of the ADC is connected to a POT meter with a fixed applied voltage. Fig 4 shows a photograph of the developed DAS system along with sensor and programmer. Fig 5 Shows a snapshot of real-time plot during data acquisition.

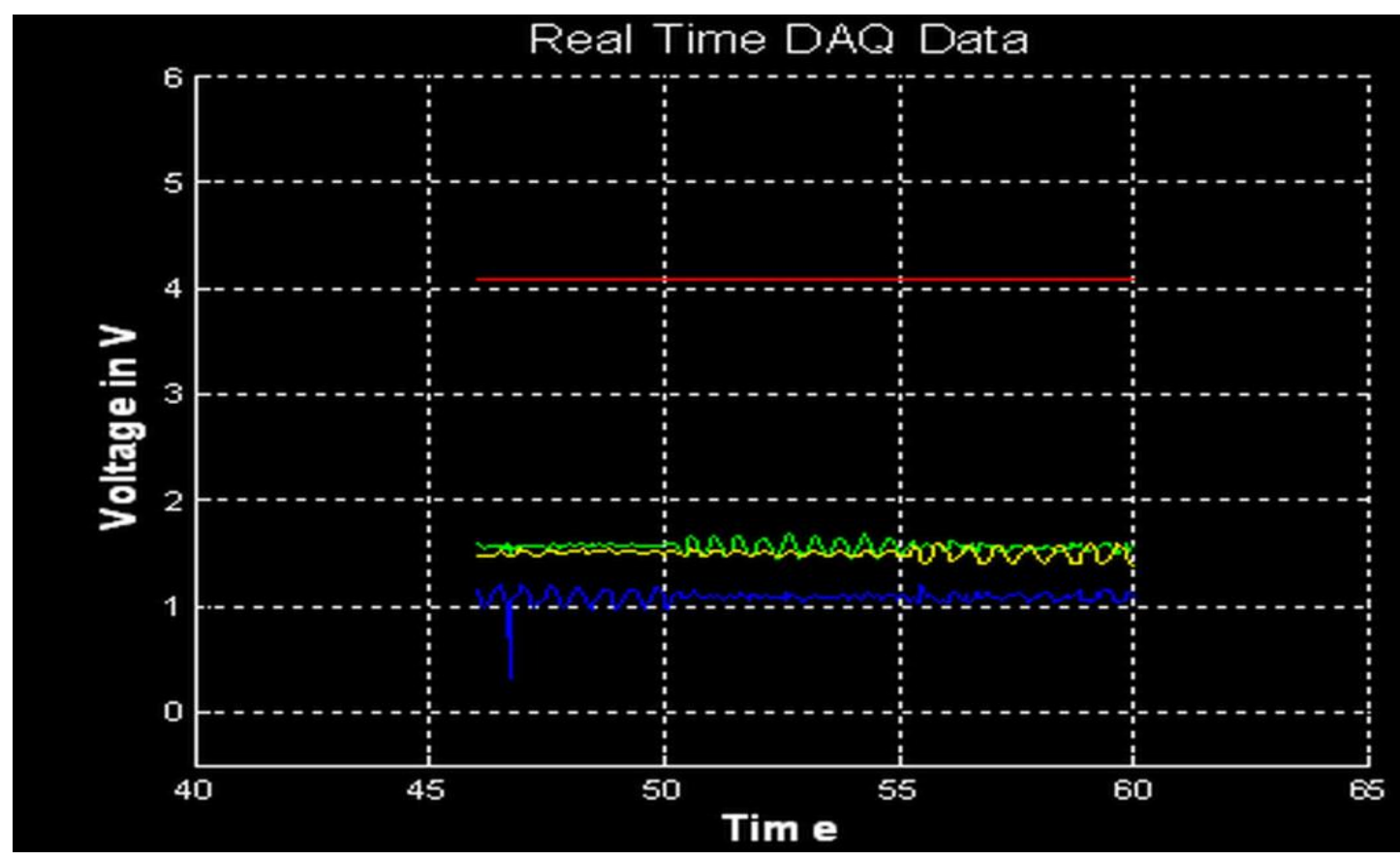

Fig 5: The waveform of the three output signal from the accelerometer. 


\section{RESULT AND DISCUSSION}

The data has been collected for $60 \mathrm{sec}$ and is being plotted in the figure window of MATLAB in real time. The acquired values are also stored in the form of a txt file. The DAS has acquires around 1282 samples in the assigned $60 \mathrm{sec}$ acquisition duration. The sample rate of the developed DAS system is found to be 21 samples per second. The plotted waveform in fig 5 shows the three output (the blue line, the yellow line and the green line in the figure window) of the accelerometer along the $\mathrm{X}$ axis, $\mathrm{Y}$ axis, and $\mathrm{Z}$ axis and the fixed value of voltage across the POT meter( the red line). Acceleration along any direction shows as a variation in the corresponding values of the accelerometer output which is clearly visible in the acquired data in the plotted figure. The fixed red line shows the constant voltage level across the POT meter.

\section{CONCLUSION}

This system can be used in the areas like inertial navigation, vibration monitoring, appliance control, and robotics application where measurement, monitoring, and storing are necessary. The designed system is low cost, compact system with 12 bit resolution having accuracy of $1.22 \mathrm{mV}$ and is also compatible to PC and laptops. It can also be interfaced to the commonly available USB port of the PC or laptop. The ADC has 13 channels hence the input of the system can be extended for data acquisition 13 different signals simultaneously. With slight modification of the software the system can also be made useful for controlling purpose using the unused digital input output pins.

\section{ACKNOWLEDGMENTS}

The authors acknowledges the unknown referees for their valuable comments and suggestions for improvement of the paper.

\section{REFERENCES}

[1] B. Nkom, H.Musa.ed,“ Development of a Novel Microcontroller based data logger", 2nd International Conference on Adaptive Science \& Technology, 2009. ICAST 2009.

[2] Ruben Posada-Gomez, Jose Jorge Enriquez-Rodriguez, Ding, Giner Alor-Hernandez, Albino Martinez-Sibaja, "USB bulk transfers between a PC and PIC microcontroller for embedded applications", CERMA '08 Proceedings of the 2008 Electronics, Robotics and Automotive Mechanics Conference.

[3] PIC18LF4553 Datasheet, available at www.microchip.com [ Last accessed $15^{\text {th }}$ September 2012]

[4] N. Monoranjan Singh, K. C. Sarma, "Design and Development of Low Cost PC Based Real Time Temperature and Humidity Monitoring System" IJECSE, Volume1, Number 3.

[5] N. Monoranjan Singh, K. C. Sarma, "Low cost PC based real time data logging system using PCs parallel port for slowly varying signals", Journal of Assam Science Society, Vol 50 (1-2), pp 36-41 (Dec 2009)

[6] N. Monoranjan Singh, K. C. Sarma, "Design of PIC 12F675 microcontroller based data acquisition system for slowly varying signals", Jl. of Instrum. Soc. of India, Vol 40 (1), pp 15-17 (March 2010).

[7] FT232R USB UART I.C Datasheet, available at www.ftdichip.com [ Last accessed $10^{\text {th }}$ September 2012 ]

[8] Jan Axelson, "Serial Port Complete - Programming and Circuits for RS-232 and RS 485 Links and Networks". 